\title{
Some Fixed Points Results in $b$-Metric and Quasi $b$-Metric Spaces
}

\author{
Reny George $\mathbb{D}^{1,2}$ Ivan D. Aranđelović $\mathbb{D}^{3},{ }^{3}$ Vesna Mišić, ${ }^{4}$ and Zoran D. Mitrovićc $\mathbb{D}^{5}$ \\ ${ }^{1}$ Department of Mathematics, College of Science and Humanities in Al-Kharj, Prince Sattam Bin Abdulaziz University, \\ Al-Kharj 11942, Saudi Arabia \\ ${ }^{2}$ Department of Mathematics and Computer Science, St. Thomas College, Bhilai, Chhattisgarh, India \\ ${ }^{3}$ University of Belgrade, Faculty of Mechanical Engineering, 11000 Beograd, Kraljice Marije 16, Serbia \\ ${ }^{4}$ Faculty of Transport and Traffic Engineering, University of East Sarajevo, Vojvode Mišića 52, \\ 53000 Doboj, Bosnia and Herzegovina \\ ${ }^{5}$ University of Banja Luka, Faculty of Electrical Engineering, 78000 Banja Luka, Patre 5, Bosnia and Herzegovina
}

Correspondence should be addressed to Reny George; renygeorge02@yahoo.com

Received 19 November 2021; Revised 30 December 2021; Accepted 5 January 2022; Published 22 January 2022

Academic Editor: Santosh Kumar

Copyright (C) 2022 Reny George et al. This is an open access article distributed under the Creative Commons Attribution License, which permits unrestricted use, distribution, and reproduction in any medium, provided the original work is properly cited.

We present a fixed point result in quasi $b$-metric spaces. Our result generalizes recent fixed point results obtained by Aleksić et al., Dung and Hang, Jovanović et al., Sarwar, and Rahman and classical results obtained by Hardy, Rogers, and Ćirić. Also, we obtain a common fixed point result in $b$-metric spaces. As a special case, we get a result of Cirić and Wong.

\section{Introduction}

The notion of a generalized contraction was presented by Ćirić in his dissertation [1]. In [1], Ćirić proved the first fixed point result for this class of mappings, which was published in [2]. Ćirić also published several papers on generalized contractions, such as for multivalued mappings in [3], on common fixed point of not necessarily commuting mappings in [4], for probabilistic metric spaces in $[5,6]$ and fixed point result of Meir-Keeler type in [7]. For further historical remarks of the papers of Cirić, see [8].

In 1973, Hardy and Rogers [9] proved a result of fixed point on metric space, which was extended to common fixed point result by Wong [10].

The results of common fixed points of Wong [10] and Ćirić [4] are independent. More concepts of common fixed points can be seen in $[11,12]$.

Also, Fréchet in the paper [13] introduced a class of metric spaces which are included in the class $b$-metric spaces. First, fixed point result in a $b$-metric space was presented by Bakhtin [14] and Czerwik [15] (for more on b-metric spaces see [16-23]). In the last few decades, many generalizations of a metric space appeared in literature. For some historical aspects of various generalizations of a metric space, the reader may refer to [24].

In this paper, we present a fixed point theorem for a mapping defined on a quasi $b$-metric space which generalizes recent fixed point results obtained by Aleksić et al. [16], Dung and Hang [18], Jovanović et al. [25], and Sarwar and Rahman [22]. Further, we obtain a result of common fixed point on a $b$-metric space. Our result generalizes the classical results presented by Ćirić [4] and Wong [10].

\section{The Quasi $b$-Metric Spaces}

We start with definition of quasi $b$-metric spaces, which was introduced by Shah and Hussain [23].

Definition 1. Let $X$ be a nonempty set, $d: X \times X \longrightarrow[0,+\infty)$ and $s \in[0,+\infty)$. Then, $(X, d, s)$ is a quasi $b$-metric space if

(1) $d(\mu, v)=0$ if and only if $\mu=v$

(2) $d(\mu, \xi) \leq s[d(\mu, v)+d(v, \xi)]$, for all $\mu, v, \xi \in X$

Clearly, $(X, d, 1)$ is a quasi metric space. 
Remark 2. Let $(X, d, s)$ be a quasi $b$-metric space and $d(\mu$, $v)=d(\nu, \mu)$ for all $\mu, v \in X$. Then, $(X, d, s)$ is a $b$-metric space.

Lemma 3. Let $(X, d, s)$ be a quasi b-metric space. Then, $s \geq 1$.

Proof. Let $\mu, v \in X$. Then, $d(\mu, v) \leq s[d(\mu, v)+d(v, v)]=s d($ $\mu, v)$. So, $s \geq 1$.

Remark 4. Let $\left(r_{n}\right)$ be a sequence of nonnegative real numbers such that $r_{n+1} \leq r_{n}$ and $\lim _{n \longrightarrow+\infty} r_{n}=0$. A quasi $b$ -metric space is a topological space with $\left\{B_{n}(\mu)\right\}_{n \in \mathbb{N}}$, as a base of neighborhood filter of the point $\mu$ where $B_{n}(\mu)=\{$ $\left.v \in X: d(\mu, v)<r_{n}\right\}$.

Definition 5. Let $(X, d, s)$ be a quasi $b$-metric space and a sequence $\left(\mu_{n}\right) \subseteq X$.

(1) Sequence $\left(\mu_{n}\right)$ is a left Cauchy sequence, if $d\left(\mu_{n}\right.$, $\left.\mu_{m}\right) \longrightarrow 0$ as $m, n \longrightarrow+\infty$

(2) A quasi $b$-metric space $(X, d, s)$ is left complete if every left Cauchy sequence converges to some $\mu \epsilon$ $X$

Definition 6. Let $(X, d, s)$ be a quasi $b$-metric space and the sequences $\left(\mu_{n}\right),\left(v_{n}\right)$ in $X$ be such that $\lim _{n \longrightarrow+\infty} \mu_{n}=\mu$ and $\lim _{m \longrightarrow+\infty} v_{n}=v$. A mapping $d$ is sequentially continuous if $\lim _{n, m \longrightarrow+\infty} d\left(\mu_{n}, v_{m}\right)=d(\mu, v)$.

We will use the following lemma in our main results.

Lemma 7 (see [26]). Let $(X, d, s)$ be a quasi b-metric space and $\left(\mu_{n}\right) \subseteq X$. If there exists $\lambda \in[0,1)$ such that

$$
d\left(\mu_{n}, \mu_{n+1}\right) \leq \lambda d\left(\mu_{n-1}, \mu_{n}\right)
$$

for all $n \in \mathbb{N}$, then $\left(\mu_{n}\right)$ is a left Cauchy sequence.

\section{A Fixed Point Theorem in Quasi $b$ - Metric Spaces}

Let $X \neq \varnothing$ and $f: X \longrightarrow X$ be a given mapping. Then, $\mu^{*} \in X$ is a fixed point of mapping $f$ if $f\left(\mu^{*}\right)=\mu^{*}$. Let $\mu_{0} \in X$, and consider the sequence $\left(\mu_{n}\right)$ defined by $\mu_{n}=f^{n}\left(\mu_{0}\right)$, i.e., $\left(\mu_{n}\right.$ ) is a sequence of Picard iterates of mapping $f$ at point $\mu_{0}$.

Now, we present our first result, which generalizes recent fixed point results obtained in $[16,18,22,25]$ for generalized contractive mappings defined on $b$-metric spaces.

Theorem 8. Let $(X, d, s)$ be a left complete quasi b-metric space and a mapping $f: X \longrightarrow X$. If there exist $\alpha, \beta, \gamma \in[0$, 1] such that $\alpha+\beta+\gamma<1, \beta \leq \gamma$ and

$$
\begin{aligned}
d(f \mu, f v) \leq & \alpha \max \left\{d(\mu, v), d(\mu, f \mu), d(v, f v), \frac{d(\mu, f v)+d(f \mu, v)}{2 s}\right\}+\beta \frac{d(\mu, f v)}{s} \\
& +\gamma d(f \mu, v),
\end{aligned}
$$

for any $\mu, v \in X$, then for any $\mu_{0} \in X$ sequence of Picard iterates $\left(\mu_{n}\right)$ defined by mapping $f$ at $\mu_{0}$ is left Cauchy sequence. Moreover, if $f$ is sequentially continuous or $d$ is sequentially continuous, then, $f$ has unique fixed point $\mu^{*} \in$ $X$ and $\mu_{n} \longrightarrow \mu^{*}$ as $n \longrightarrow+\infty$.

Proof. Let $\mu_{0} \in X$ be arbitrary and $\left(\mu_{n}\right)$ sequence of Picard iterates defined by $f$ at $\mu_{0}$. Then

$$
\begin{aligned}
& d\left(\mu_{n+1}, \mu_{n+2}\right)=d\left(f \mu_{n}, f \mu_{n+1}\right) \\
& \leq \alpha \max \left\{d\left(\mu_{n}, \mu_{n+1}\right), d\left(\mu_{n}, f \mu_{n}\right), d\left(\mu_{n+1}, f \mu_{n+2}\right), \frac{d\left(\mu_{n}, f \mu_{n+1}\right)+d\left(f \mu_{n}, \mu_{n+1}\right)}{2 s}\right\} \\
&+\beta \frac{d\left(\mu_{n}, f \mu_{n+1}\right)}{s}+\gamma d\left(f \mu_{n}, \mu_{n+1}\right) \\
& \leq \alpha \max \left\{d\left(\mu_{n}, \mu_{n+1}\right), d\left(\mu_{n+1}, \mu_{n+2}\right), \frac{d\left(\mu_{n}, \mu_{n+1}\right)+d\left(\mu_{n+1}, \mu_{n+2}\right)}{2}\right\}+\beta \frac{d\left(\mu_{n}, \mu_{n+2}\right)}{s} \\
& \leq \alpha \max \left\{d\left(\mu_{n}, \mu_{n+1}\right), d\left(\mu_{n+1}, \mu_{n+2}\right)\right\}+\beta d\left(\mu_{n}, \mu_{n+1}\right)+\beta d\left(\mu_{n+1}, \mu_{n+2}\right) .
\end{aligned}
$$

If $d\left(\mu_{n}, \mu_{n+1}\right)<d\left(\mu_{n+1}, \mu_{n+2}\right)$, then,

$$
(1-\alpha-\beta) d\left(\mu_{n+1}, \mu_{n+2}\right)<\beta d\left(\mu_{n}, \mu_{n+1}\right)
$$

which implies

$$
d\left(\mu_{n+1}, \mu_{n+2}\right) \leq \frac{\beta}{1-\alpha-\beta} d\left(\mu_{n}, \mu_{n+1}\right)<d\left(\mu_{n}, \mu_{n+1}\right) .
$$

So, $d\left(\mu_{n}, \mu_{n+1}\right) \geq d\left(\mu_{n+1}, \mu_{n+2}\right)$ which implies

$$
(1-\beta) d\left(\mu_{n+1}, \mu_{n+2}\right) \leq(\alpha+\beta) d\left(\mu_{n}, \mu_{n+1}\right) .
$$

Hence, we get that

$d\left(\mu_{n+1}, \mu_{n+2}\right) \leq \frac{\alpha+\beta}{1-\beta} d\left(\mu_{n}, \mu_{n+1}\right)<\frac{\alpha+\beta}{\alpha+\gamma} d\left(\mu_{n}, \mu_{n+1}\right)=\lambda d\left(\mu_{n}, \mu_{n+1}\right)$,

where $\lambda=\alpha+\beta / \alpha+\gamma<1$. So, by Lemma 7 , we obtain that $\left(\mu_{n}\right)$ is left Cauchy sequence. It is convergent because $(X, d$ $, s)$ is left complete. Thus, exists $\mu^{*} \in X$ such that $\mu^{*}=\lim$ $\mu_{n}$

Case 9. Let a mapping $f$ be a sequentially continuous. Then

$$
\mu^{*}=\lim \mu_{n}=\lim f \mu_{n}=f \mu^{*} .
$$

Case 10. Let $d$ be a sequentially continuous.

Then

$$
\begin{aligned}
d\left(f \mu_{n}, f \mu^{*}\right) \leq & \alpha \max \left\{d\left(\mu_{n}, \mu^{*}\right), d\left(\mu_{n}, \mu_{n+1}\right), d\left(\mu^{*}, f \mu^{*}\right), \frac{d\left(\mu_{n}, f \mu^{*}\right)+d\left(f \mu_{n}, \mu^{*}\right)}{2 s}\right\} \\
& +\beta \frac{d\left(\mu_{n}, \mu_{n+2}\right)}{2 s}+\gamma d\left(f \mu_{n}, \mu^{*}\right),
\end{aligned}
$$


which implies

$$
\begin{aligned}
\lim d\left(f \mu_{n}, f \mu^{*}\right) \leq & \lim \left[\alpha\left\{d\left(\mu_{n}, \mu^{*}\right), d\left(\mu^{*}, f \mu^{*}\right), \frac{d\left(\mu_{n}, f \mu^{*}\right)+d\left(f \mu_{n}, \mu^{*}\right)}{2 s}\right\}\right. \\
& \left.+\beta \frac{d\left(\mu_{n}, \mu_{n+2}\right)}{2 s}+\gamma d\left(f \mu_{n}, \mu^{*}\right)\right] .
\end{aligned}
$$

So, we get that

$$
\begin{aligned}
\left.d\left(\lim \mu_{n+1}, f \mu^{*}\right)\right) \leq & \lim \left[\alpha \operatorname { m a x } \left\{d\left(\lim \mu_{n}, \mu^{*}\right), d\left(\lim \mu_{n}, \lim \mu_{n+1}\right), d\left(\mu^{*}, f \mu^{*}\right),\right.\right. \\
& \left.\cdot \frac{d\left(\lim \mu_{n}, f \mu^{*}\right)+d\left(\lim \mu_{n+1}, \mu^{*}\right)}{2 s}\right\}+\beta \frac{d\left(\mu_{n}, \mu_{n+2}\right)}{2 s} \\
& \left.+\gamma d\left(\lim \mu_{n+1}, \mu^{*}\right)\right] .
\end{aligned}
$$

Hence,

$$
\begin{aligned}
d\left(\mu^{*}, f \mu^{*}\right) \leq & \alpha \max \left\{d\left(\mu^{*}, \mu^{*}\right), d\left(\mu^{*}, \mu^{*}\right), d\left(\mu^{*}, f \mu^{*}\right), \frac{d\left(\mu^{*}, f \mu^{*}\right)+d\left(\mu^{*}, \mu^{*}\right)}{2 s}\right\} \\
& +\beta \frac{d\left(\mu^{*}, \mu^{*}\right)}{2 s}+\gamma d\left(\mu^{*}, \mu^{*}\right)=\alpha d\left(\mu^{*}, f \mu^{*}\right) .
\end{aligned}
$$

It follows that $\mu^{*}=f\left(\mu^{*}\right)$ because $\alpha \in[0,1)$. Finally, suppose that there are two fixed points of mapping $f$, i.e., $f \mu^{*}$ $=\mu^{*}, f v^{*}=v^{*}$. Then, we get

$$
\begin{aligned}
d\left(\mu^{*}, v^{*}\right)= & d\left(f \mu^{*}, f v^{*}\right) \leq \alpha\left\{d\left(\mu^{*}, v^{*}\right), d\left(\mu^{*}, \mu^{*}\right), d\left(v^{*}, v^{*}\right), \frac{d\left(\mu^{*}, v^{*}\right)+d\left(\mu^{*}, v^{*}\right)}{2 s}\right\} \\
& +\beta \frac{d\left(\mu^{*}, v^{*}\right)}{2 s}+\gamma d\left(\mu^{*}, v^{*}\right) \leq(\alpha+\beta+\gamma) d\left(\mu^{*}, v^{*}\right) .
\end{aligned}
$$

which implies that $\mu^{*}=v^{*}$.

Corollary 11. Let $(X, d, s)$ be a left complete quasi b-metric space and a mapping $f: X \longrightarrow X$. If there exist $\alpha \in[0,1)$ such that

$$
d(f \mu, f v) \leq \alpha d(\mu, v)
$$

for any $\mu, v \in X$, then for any $\mu_{0} \in X$ sequence of Picard iterates $\left(\mu_{n}\right)$ defined by mapping $f$ at $\mu_{0}$ is left Cauchy sequence. Moreover, if $f$ is sequentially continuous or $d$ is sequentially continuous, then, $f$ has unique fixed point $\mu^{*} \epsilon$ $X$ and $\mu_{n} \longrightarrow \mu^{*}$ as $n \longrightarrow+\infty$.

Example 12. Let $X=[0,1]$ and mapping $f: X \longrightarrow X$ defined by $f \mu=\mu / 2, \mu \in X$. Let $d: X \times X \longrightarrow[0,+\infty)$ defined by

$$
d(\mu, v)= \begin{cases}(\mu-v)^{2}, & \mu>v \\ (\mu-v)^{4}, & \mu<v \\ 0, & \mu=v .\end{cases}
$$

Since, $(a+b)^{2} \leq 2\left(a^{2}+b^{2}\right)$ and $(a+b)^{4} \leq 8\left(a^{4}+b^{4}\right)$, for all $a, b \in \mathbb{R}$, we obtain that for $d$ holds

$$
d(\mu, \xi) \leq 8[d(\mu, v)+d(\nu, \xi)]
$$

for all $\mu, v, \xi \in X$. Also, $d(\mu, v)=0$ if and only if $\mu=v$. So, $(X, d, 8)$ is a quasi $b$-metric space. Note that $d(\mu, v)=d(v$, $\mu)$ does not hold in the general case. In this case, all the conditions of Corollary 11 are valid, and we conclude that the mapping $f$ has a fixed point.

\section{A Common Fixed Point Theorem in $b$ - Metric Spaces}

Now we obtain a common fixed point result for mappings defined on $b$-metric spaces. Our result improves the classical results presented by Ćirić [4] and Wong [10].

Theorem 13. Let $(X, d, s)$ be a complete b-metric space and the mappings $f, g: X \longrightarrow X$. If there exist $\alpha, \beta \in[0,1]$ such that $\alpha+2 \beta<1$ and

$$
\begin{aligned}
d(f \mu, g v) \leq & \alpha \max \left\{d(\mu, v), d(\mu, f \mu), d(v, g v), \frac{d(\mu, g v)+d(f \mu, v)}{2 s}\right\}+\beta \frac{d(\mu, g v)}{s} \\
& +\beta \frac{d(f \mu, v)}{s},
\end{aligned}
$$

for any $\mu, \nu \in X$, then for any $\mu_{0} \in X$ sequence of Picard iterates $\left(\mu_{n}\right)$ defined by $g \circ f$ at $\mu_{0}$ is left Cauchy sequence. If $f$ and $g$ are sequentially continuous or $d$ is sequentially continuous then $f$ and $g$ has unique fixed point which is unique limit of all Picard sequences defined by $g \circ f$.

Proof. Let $\mu_{0} \in X$ be arbitrary and $\left(\mu_{n}\right)$ sequence defined by $\mu_{2 n+1}=f \mu_{2 n}$ and $\mu_{2 n+2}=g \mu_{2 n+1}$. Then

$$
\begin{aligned}
d\left(\mu_{2 n+1}, \mu_{2 n+2}\right)= & d\left(f \mu_{2 n}, g \mu_{2 n+1}\right) \leq \alpha \max \left\{d\left(\mu_{2 n}, \mu_{2 n+1}\right), d\left(\mu_{2 n}, f \mu_{2 n}\right), d\left(\mu_{2 n+1}, g \mu_{2 n+1}\right),\right. \\
& \left.\cdot \frac{d\left(\mu_{2 n}, g \mu_{2 n+1}\right)+d\left(\mu_{2 n}, \mu_{2 n+1}\right)}{2 s}\right\}+\beta \frac{d\left(\mu_{2 n}, f \mu_{n+1}\right)}{s}+\beta \frac{d\left(f \mu_{2 n}, \mu_{2 n+1}\right)}{s} \\
\leq & \alpha \max \left\{d\left(\mu_{2 n}, \mu_{2 n+1}\right), d\left(\mu_{2 n+1}, \mu_{2 n+2}\right), \frac{d\left(\mu_{2 n}, \mu_{2 n+1}\right)+d\left(\mu_{2 n+1}, \mu_{2 n+2}\right)}{2}\right\} \\
& +\beta \frac{d\left(\mu_{2 n}, \mu_{n+2}\right)}{s} \leq \alpha \max \left\{d\left(\mu_{2 n}, \mu_{2 n+1}\right), d\left(\mu_{2 n+1}, \mu_{2 n+2}\right)\right\} \\
& +\beta d\left(\mu_{2 n}, \mu_{2 n+1}\right)+\beta d\left(\mu_{2 n+1}, \mu_{2 n+2}\right) .
\end{aligned}
$$

If $d\left(\mu_{2 n}, \mu_{2 n+1}\right)<d\left(\mu_{2 n+1}, \mu_{n+2}\right)$ then

$$
(1-\alpha-\beta) d\left(\mu_{2 n+1}, \mu_{2 n+2}\right)<\beta d\left(\mu_{2 n}, \mu_{2 n+1}\right) .
$$

So, we get that

$$
d\left(\mu_{2 n+1}, \mu_{2 n+2}\right) \leq \frac{\beta}{1-\alpha-\beta} d\left(\mu_{2 n}, \mu_{2 n+1}\right)<d\left(\mu_{2 n}, \mu_{2 n+1}\right),
$$

therefore, $d\left(\mu_{2 n}, \mu_{2 n+1}\right) \geq d\left(\mu_{2 n+1}, \mu_{2 n+2}\right)$ which implies

$$
(1-\beta) d\left(\mu_{n+1}, \mu_{n+2}\right) \leq(\alpha+\beta) d\left(\mu_{2 n}, \mu_{2 n+1}\right) .
$$


Hence, we get that

$$
d\left(\mu_{2 n+1}, \mu_{2 n+2}\right) \leq \frac{\alpha+\beta}{1-\beta} d\left(\mu_{2 n}, \mu_{2 n+1}\right) .
$$

So, we obtained

$$
d\left(\mu_{2 n+1}, \mu_{2 n+2}\right) \leq \lambda d\left(\mu_{2 n}, \mu_{2 n+1}\right)
$$

where $\lambda=\alpha+\beta / 1-\beta<1$. Further, we have

$$
\begin{aligned}
d\left(\mu_{2 n}, \mu_{2 n+1}\right)= & d\left(g \mu_{2 n-1}, f \mu_{2 n}\right)=d\left(f \mu_{2 n}, g \mu_{2 n-1}\right) \\
\leq & \alpha \max \left\{d\left(\mu_{2 n-1}, \mu_{2 n}\right), d\left(\mu_{2 n}, f \mu_{2 n}\right), d\left(\mu_{2 n-1}, g \mu_{2 n-1}\right),\right. \\
& \left.\cdot \frac{d\left(\mu_{2 n-1}, f \mu_{2 n}\right)+d\left(\mu_{2 n}, g \mu_{2 n-1}\right)}{2 s}\right\}+\beta \frac{d\left(\mu_{2 n}, g \mu_{2 n-1}\right)}{s}+\beta \frac{d\left(f \mu_{2 n}, \mu_{2 n-1}\right)}{s} \\
\leq & \alpha \max \left\{d\left(\mu_{2 n-1}, \mu_{2 n}\right), d\left(\mu_{2 n}, \mu_{2 n+1}\right), \frac{d\left(\mu_{2 n}, \mu_{2 n+1}\right)+d\left(\mu_{2 n-1}, \mu_{2 n}\right)}{2}\right\} \\
& +\beta \frac{d\left(\mu_{2 n-1}, \mu_{2 n+1}\right)}{s} \leq \alpha \max \left\{d\left(\mu_{2 n}, \mu_{2 n+1}\right), d\left(\mu_{2 n+1}, \mu_{2 n+2}\right)\right\} \\
& +\beta d\left(\mu_{2 n-1}, \mu_{2 n}\right)+\beta d\left(\mu_{2 n}, \mu_{2 n+1}\right) .
\end{aligned}
$$

If $d\left(\mu_{2 n-1}, \mu_{2 n}\right)<d\left(\mu_{n}, \mu_{n+1}\right)$ then

$$
(1-\alpha-\beta) d\left(\mu_{2 n}, \mu_{2 n+1}\right)<\beta d\left(\mu_{2 n-1}, \mu_{2 n}\right),
$$

which implies

$$
d\left(\mu_{2 n}, \mu_{2 n+1}\right) \leq \frac{\beta}{1-\alpha-\beta} d\left(\mu_{2 n}, \mu_{2 n-1}\right)<d\left(\mu_{2 n-1}, \mu_{2 n}\right),
$$

therefore, $d\left(\mu_{2 n-1}, \mu_{2 n}\right) \geq d\left(\mu_{2 n}, \mu_{2 n+1}\right)$ which implies that

$$
d\left(\mu_{2 n}, \mu_{2 n+1}\right) \leq \alpha d\left(\mu_{2 n-1}, \mu_{2 n}\right)+\beta d\left(\mu_{2 n-1}, \mu_{2 n}\right)+\beta d\left(\mu_{2 n}, \mu_{2 n+1}\right) .
$$

Therefore, we obtain

$$
(1-\beta) d\left(\mu_{2 n}, \mu_{2 n+1}\right) \leq(\alpha+\beta) d\left(\mu_{2 n-1}, \mu_{2 n}\right) .
$$

It follows

$$
d\left(\mu_{2 n}, \mu_{2 n+1}\right) \leq \frac{\alpha+\beta}{1-\beta} d\left(\mu_{2 n-1}, \mu_{2 n}\right) .
$$

So we obtain,

$$
d\left(\mu_{2 n}, \mu_{2 n+1}\right) \leq \lambda d\left(\mu_{2 n-1}, \mu_{2 n}\right),
$$

where $\lambda=\alpha+\beta / 1-\beta<1$. Hence,

$$
d\left(\mu_{n}, \mu_{n+1}\right) \leq \lambda d\left(\mu_{n-1}, \mu_{n}\right),
$$

for each positive integer $n$. So, by Lemma 7, we obtain that $\left(\mu_{n}\right)$ is a Cauchy sequence. It is convergent because $(X, d, s$ ) is complete. Therefore, there exists $\xi \in X$ such that $\xi=$ $\lim \mu_{n}$.
Case 14. Let $f$ and $g$ be sequentially continuous functions. Then, we have

$$
\xi=\lim \mu_{n}=\lim f \mu_{n}=f \xi=\lim \mu_{n}=\lim g \mu_{n}=g \xi .
$$

Case 15. Let $d$ be a sequentially continuous. Then,

$$
\begin{aligned}
& d\left(f \xi, g \mu_{2 n+1}\right) \\
& \leq \alpha \max \left\{d\left(\xi, \mu_{2 n+1}\right), d(\xi, f \xi), d\left(\mu_{2 n+1}, g \mu_{2 n+1}\right), \frac{d\left(\mu_{2 n+1}, f \xi\right)+d\left(g \mu_{2 n+1}, \xi\right)}{2 s}\right\} \\
& \quad+\beta \frac{d\left(\mu_{2 n+1}, f \xi\right)}{2 s}+\beta \frac{d\left(g \mu_{2 n+1}, \xi\right)}{s},
\end{aligned}
$$

which implies

$$
\begin{aligned}
\lim d\left(f \xi, g \mu_{2 n+1}\right) \leq & \lim \left[\alpha \operatorname { m a x } \left\{d\left(\xi, \mu_{2 n+1}\right), d(\xi, f \xi), d\left(\mu_{2 n+1}, g \mu_{2 n+1}\right),\right.\right. \\
& \left.\cdot \frac{d\left(\mu_{2 n+1}, f \xi\right)+d\left(g \mu_{2 n+1}, \xi\right)}{2 s}\right\}+\beta \frac{d\left(\mu_{2 n+1}, f \xi\right)}{2 s} \\
& \left.+\beta \frac{d\left(g \mu_{2 n+1}, \xi\right)}{s}\right] .
\end{aligned}
$$

So, we get that

$$
\begin{aligned}
d\left(\lim g \mu_{2 n+1}, f \xi\right) \leq & \alpha \max \left\{d\left(\lim \mu_{2 n+1}, \xi\right) d(\xi, f \xi), d\left(\lim \mu_{2 n+1}, \lim f \mu_{2 n+1}\right),\right. \\
& \left.\cdot \frac{d\left(\lim \mu_{2 n+1}, f \xi\right)+d\left(\lim g \mu_{2 n+1}, \xi\right)}{2 s}\right\}+\beta \frac{d\left(\lim \mu_{2 n+1}, f \xi\right)}{2 s} \\
& \left.+\beta \frac{d\left(\lim g \mu_{2 n+1}, \xi\right)}{s}\right] .
\end{aligned}
$$

Hence,

$$
\begin{aligned}
d(\xi, f \xi) & \leq \alpha \max \left\{d(\xi, \xi), d(\xi, f \xi), d(\xi, \xi), \frac{d(\xi, f \xi)+d(\xi, \xi)}{2 s}\right\}+\beta \frac{d(\xi, f \xi)}{2 s}+\beta d(\xi, \xi) \\
& <(\alpha+\beta) d(\xi, f \xi) .
\end{aligned}
$$
have

It follows that $\xi=f \xi$ because $(\alpha+\beta) \in[0,1)$. Further, we

$$
\begin{aligned}
d\left(f \mu_{2 n}, g \xi\right) \leq & \alpha \max \left\{d\left(\mu_{2 n}, \xi\right), d\left(\mu_{2 n}, f \mu_{2 n}\right), d(\xi, g \xi), \frac{d\left(\mu_{2 n}, g \xi\right)+d\left(f \mu_{2 n}, \xi\right)}{2 s}\right\} \\
& +\beta \frac{d\left(\mu_{2 n}, g \xi\right)}{2 s}+\beta \frac{d\left(f \mu_{2 n}, \xi\right)}{s},
\end{aligned}
$$

which implies

$$
\begin{aligned}
\lim d\left(f \mu_{2 n}, g \xi\right) \leq & \lim \left[\alpha \max \left\{d\left(\mu_{2 n}, \xi\right), d\left(\mu_{2 n}, f \mu_{2 n}\right), d(\xi, g \xi), \frac{d\left(\mu_{2 n}, g \xi\right)+d\left(f \mu_{2 n}, \xi\right)}{2 s}\right\}\right. \\
& \left.+\beta \frac{d\left(\mu_{2 n}, g \xi\right)}{2 s}+\beta \frac{d\left(f \mu_{2 n}, \xi\right)}{s}\right] .
\end{aligned}
$$


So, we get that

$$
\begin{aligned}
d\left(\lim f \mu_{2 n}, g \xi\right) \leq & \alpha \max \left\{d\left(\lim \mu_{2 n}, \xi\right), d\left(\lim \mu_{2 n}, \lim f \mu_{2 n}\right), d(\xi, g \xi),\right. \\
& \left.\cdot \frac{d\left(\lim \mu_{2 n}, g \xi\right)+d\left(\lim f \mu_{2 n}, \xi\right)}{2 s}\right\}+\beta \frac{d\left(\lim \mu_{2 n}, g \xi\right)}{2 s} \\
& \left.+\beta \frac{d\left(\lim f \mu_{2 n}, \xi\right)}{s}\right] .
\end{aligned}
$$

Hence,

$$
\begin{aligned}
d(\xi, g \xi) & \leq \alpha \max \left\{d(\xi, \xi), d(\xi, \xi), d(\xi, g \xi), \frac{d(\xi, g \xi)+d(\xi, \xi)}{2 s}\right\}+\beta \frac{d(\xi, g \xi)}{2 s}+\beta d(\xi, \xi) \\
& <(\alpha+\beta) d(\xi, g \xi) .
\end{aligned}
$$

It follows that $\xi=g(\xi)$ because $(\alpha+\beta) \in[0,1)$.

Now, we prove that the fixed point is unique. Suppose that there are $\xi$ and $\xi^{\prime}$, i.e., $g \xi=f \xi=\xi$ and $g \xi^{\prime}=f \xi^{\prime}=\xi^{\prime}$. Then, we obtain

$$
\begin{aligned}
d\left(\xi, \xi^{\prime}\right)= & d\left(f \xi, \xi^{\prime}\right) \leq \alpha\left\{d\left(\xi, \xi^{\prime}\right), d(\xi, \xi) d\left(\xi^{\prime}, \xi^{\prime}\right), \frac{d\left(\xi, \xi^{\prime}\right)+d\left(\xi, \xi^{\prime}\right)}{2 s}\right\} \\
& +\beta \frac{d\left(\xi, \xi^{\prime}\right)}{2 s}+\beta d\left(\xi, \xi^{\prime}\right) \leq(\alpha+2 \beta) d\left(\xi, \xi^{\prime}\right) .
\end{aligned}
$$

which implies that $\xi=\xi^{\prime}$.

Corollary 16. Let $(X, d, s)$ be a complete b-metric space and mapping $f: X \longrightarrow X$. If there exist $\alpha, \beta, \gamma \in[0,1]$ such that $\alpha+\beta+\gamma<1$ and

$$
\begin{aligned}
d(f \mu, f v) \leq & \alpha \max \left\{d(\mu, v), d(\mu, f \mu), d(v, f v), \frac{d(\mu, f v)+d(f \mu, v)}{2 s}\right\} \\
& +\beta \frac{d(\mu, f v)}{s}+\gamma \frac{d(f \mu, v)}{s},
\end{aligned}
$$

for any $\mu, v \in X$, then for any $\mu_{0} \in X$ sequence of Picard iterates $\left(\mu_{n}\right)$ defined by $f$ at $\mu_{0}$ is Cauchy sequence. If $f$ is sequentially continuous or $d$ is sequentially continuous, then, $f$ has unique fixed point which is unique limit of all Picard sequences defined by $f$.

Proof. From

$$
\begin{aligned}
d(f \mu, f v) \leq & \alpha \max \left\{d(\mu, v), d(\mu, f \mu), d(v, f v), \frac{d(\mu, f v)+d(f \mu, v)}{2 s}\right\} \\
& +\beta \frac{d(\mu, f v)}{s}+\gamma \frac{d(f \mu, v)}{s},
\end{aligned}
$$

and

$$
\begin{aligned}
d(f \nu, f \mu) \leq & \alpha \max \left\{d(\nu, \mu), d(\nu, f v), d(\mu, f \mu), \frac{d(\nu, f \mu)+d(f v, \mu)}{2 s}\right\} \\
& +\beta \frac{d(\nu, f \mu)}{s}+\gamma \frac{d(f v, \mu)}{s}
\end{aligned}
$$

it follows

$$
\begin{aligned}
d(f \mu, f v) \leq & \alpha \max \left\{d(\mu, v), d(\mu, f \mu), d(v, f v), \frac{d(\mu, f v)+d(f \mu, v)}{2 s}\right\} \\
& +\delta \frac{d(\mu, f v)}{s}+\delta \frac{d(f \mu, v)}{s}
\end{aligned}
$$

where $\delta=\beta+\gamma / 2$

Example 17. Let $X=[0,4]$ and $d(\mu, v)=(\mu-v)^{2}$, for each $\mu$ ,$v \in X$. Then $(X, d, 2)$ is a $b$-metric space. Define a mapping $f: X \longrightarrow X$ by

$$
f(t)= \begin{cases}\frac{t}{3}, & t \in[0,3], \\ \frac{t}{6}, & t \in(3,4],\end{cases}
$$

for any $t \in X$. For $\mu, v \in[0,3]$, we have

$$
d(f \mu, f v)=\frac{1}{9}(\mu-v)^{2} .
$$

For $\mu, v \in(3,4]$, we have

$$
d(f \mu, f v)=\frac{1}{36} d(\mu, v) .
$$

For $\mu \in[0,3]$ and $v \in(3,4]$, we have

$$
d(f \mu, f v)=\left(\frac{\mu}{3}-\frac{v}{6}\right)^{2} \leq \frac{4}{9}<\frac{1}{2} d(v, f v)
$$

because $d(v, f v)=(5 v / 6)^{2}>25 \cdot 9 / 36$.

For $v \in[0,3]$ and $\mu \in(3,4]$, we have

$$
d(f \mu, f v)=\left(\frac{\mu}{3}-\frac{v}{6}\right)^{2} \leq \frac{16}{9}<\frac{3}{4} d(\mu, f \mu)
$$

because $d(\mu, f \mu)=(5 \mu / 6)^{2}>25 \cdot 9 / 36$.

Since conditions of Corollary 16 is satisfied for $\alpha=3 / 4$ and $\beta=\gamma=0$. So, $f$ has unique fixed point which is unique limit of all Picard sequences defined by $f$, because $d$ is sequentially continuous.

\section{Data Availability}

No data are used. 


\section{Conflicts of Interest}

The authors declare that they have no conflicts of interest.

\section{References}

[1] L. B. Ćirić, "Fixed and periodic points of contractive operators, [Ph.D. thesis]," Univ. Belgrade-Faculty of Siences, Serbian, 1970.

[2] L. B. Ćirić, "Generalized Contractions and Fixed-Point Theorems," Publications de l'Institut Mathématiqu, vol. 12, no. 26, pp. 19-26, 1971.

[3] L. B. Ćirić, "Fixed points for generalized multi-valued contractions," Matematichki Vesnik, vol. 9, no. 24, pp. 265-272, 1972.

[4] L. B. Ćirić, "On a family of contractive maps and fixed points," Institut Mathématique, vol. 17, no. 31, pp. 45-51, 1974.

[5] L. B. Cirić, On Fixed Points of Contractive Mappings on Probabilistic Metric Spaces, 5. Balcan mathematical congres, Book of Abstracts, Beograd, 1974.

[6] L. B. Cirić, "On fixed points of generalized contractions on probabilistic metric spaces," Institut Mathématique, vol. 18, no. 32, pp. 71-78, 1975.

[7] L. B. Cirić, "Fixed points of weakly contraction mappings," Institut Mathématique, vol. 20, no. 34, pp. 79-84, 1976.

[8] I. D. Arandelović and M. S. Mateljević, "Ljubomir B. Ćirić (1935-2016)," Filomat, vol. 31, no. 11, pp. 3035-3040, 2017.

[9] G. E. Hardy and T. D. Rogers, "A generalization of a fixed point theorem of Reich," Canadian Mathematical Bulletin, vol. 16, no. 2, pp. 201-206, 1973.

[10] C. S. Wong, "Common fixed points of two mappings," Pacific Journal of Mathematics, vol. 48, no. 1, pp. 299-312, 1973.

[11] D. Gopal and R. K. Bisht, "Metrical Common Fixed Points and Commuting Type Mappings," in Background and Recent Developments of Metric Fixed Point Theory, D. Gopal, Ed., pp. 29-67, CRC Press, Boca Raton, FL, USA, 2018.

[12] D. K. Patel, P. Kumam, and D. Gopal, "Some discussion on the existence of common fixed points for a pair of maps," Fixed Point Theory and Applications, vol. 2013, 2013.

[13] M. Fréchet, "Sur quelques points du calcul fonctionnel," Rendiconti del Circolo Matematico di Palermo, vol. 22, pp. 1-74, 1906.

[14] I. A. Bakhtin, "The contraction mapping principle in quasimetric spaces," Ulyanovsk State Pedagogical University (Ul'yanovskî̂ Gosudarstvennyî Pedagogicheskî̂ Institut Im. I. N. Ul'yanova), Ulyanovsk, vol. 30, pp. 26-37, 1989.

[15] S. Czerwik, "Contraction mappings in b-metric spaces," Acta mathematica et informatica universitatis ostraviensis, vol. 1, pp. 5-11, 1993.

[16] S. Aleksić, H. Huang, Z. D. Mitrović, and S. Radenović, "Remarks on some fixed point results in $b$-metric spaces," Journal of Fixed Point Theory and Applications, vol. 20, no. 4, p. 147, 2018.

[17] M. U. Ali, H. Aydi, and M. Alansari, "New generalizations of set valued interpolative Hardy-Rogers type contractions in $b$ -metric spaces," Journal of Function Spaces, vol. 2021, Article ID 6641342, 8 pages, 2021.

[18] N. V. Dung and V. T. L. Hang, "On relaxations of contraction constants and Caristi's theorem in b-metric spaces," Journal of Fixed Point Theory and Applications, vol. 19, pp. 257-284, 2016.
[19] K. Javed, F. Uddin, H. Aydi, A. Mukheimer, and M. Arshad, "Ordered-theoretic fixed point results in fuzzy b-metric spaces with an application," Journal of Mathematics, vol. 2021, Article ID 6663707, 7 pages, 2021.

[20] K. Javed, H. Aydi, F. Uddin, and M. Arshad, "On orthogonal partial $b$-metric spaces with an application," Journal of Mathematics, vol. 2021, Article ID 6692063, 7 pages, 2021.

[21] R. Miculescu and A. Mihail, "New fixed point theorems for setvalued contractions in b-metric spaces," Journal of Fixed Point Theory and Applications, vol. 19, pp. 2153-2163, 2017.

[22] M. Sarwar and M. U. Rahman, "Fixed point theorems for Ciric's and generalized contractions in b-metric spaces," International Journal of Analysis and Applications, vol. 7, no. 1, pp. 70-78, 2015.

[23] M. H. Shah and N. Hussain, "Nonlinear contractions in partially ordered quasi b-metric spaces," Korean Mathematical Society, vol. 27, no. 1, pp. 117-128, 2012.

[24] D. Gopal and P. Agarwal, MMetric Structures and Fixed Point Theory, P. Kumam, Ed., Chapman and Hall/CRC, 1st edition, 2021.

[25] M. Jovanović, Z. Kadelburg, and S. Radenović, "Common fixed point results in metric type spaces," Fixed Point Theory and Applications, vol. 2010, Article ID 978121, 15 pages, 2010.

[26] Z. D. Mitrović, I. Aranđelović, V. Mišić, H. Aydi, and B. Samet, "On $\mathrm{d} *$-complete topological spaces and related fixed point results," Mathematics, vol. 8, article 1447, 2020. 